\title{
Filigrane
}

Écoutes psychothérapiques

\section{Aux limites de la temporalité : l’archaïque et la mort}

\section{Marc Bonnet}

Volume 22, numéro 1, printemps 2013

Psychanalyse et temporalités

URI : https://id.erudit.org/iderudit/1017346ar

DOI : https://doi.org/10.7202/1017346ar

Aller au sommaire du numéro

Éditeur(s)

Revue Santé mentale au Québec

ISSN

1192-1412 (imprimé)

1911-4656 (numérique)

Découvrir la revue

Citer cet article

Bonnet, M. (2013). Aux limites de la temporalité : l'archaïque et la mort.

Filigrane, 22(1), 53-67. https://doi.org/10.7202/1017346ar

\section{Résumé de l'article}

Dans ce travail, nous traitons des deux limites de la temporalité que sont l'archaïque d'une part et la mort d'autre part. Le chemin parcouru entre les deux constitue le projet identificatoire. L'archaïque s'organise sur l'espace originaire et s'illustre selon deux potentialités que sont la potentialité mélancolique et la potentialité paranoïaque. La mort demeure une sérieuse énigme pour le sujet dont les contours sont peut-être en train de se modifier à l'heure actuelle. Le projet identificatoire se structure sur la base de l'identification primaire à double composante d'identification symbolique, qui préexiste au sujet, et d'identification narcissique, qui en marque l'origine historique. Notre propos se termine par un questionnement sur la problématique ouverte par les nouveaux processus de subjectivation.
Ce document est protégé par la loi sur le droit d'auteur. L'utilisation des services d'Érudit (y compris la reproduction) est assujettie à sa politique d'utilisation que vous pouvez consulter en ligne.

https://apropos.erudit.org/fr/usagers/politique-dutilisation/ 


\section{Aux limites de la temporalité: l'archaïque et la mort}

\section{Marc Bonnet}

Dans ce travail, nous traitons des deux limites de la temporalité que sont l'archaïque d'une part et la mort d'autre part. Le chemin parcouru entre les deux constitue le projet identificatoire. L'archaïque s'organise sur l'espace originaire et s'illustre selon deux potentialités que sont la potentialité mélancolique et la potentialité paranoïaque. La mort demeure une sérieuse énigme pour le sujet dont les contours sont peut-être en train de se modifier à I'heure actuelle. Le projet identificatoire se structure sur la base de l'identification primaire à double composante d'identification symbolique, qui préexiste au sujet, et d'identification narcissique, qui en marque l'origine historique. Notre propos se termine par un questionnement sur la problématique ouverte par les nouveaux processus de subjectivation.

\section{Avant-propos}

ans le présent essai, je m’intéresserai aux deux bornes qui marquent la temporalité de la vie humaine, à savoir l'archaïque et la mort, pour essayer de déterminer ce qui les caractérise, mais aussi ce qui les rapproche et ce qui les différencie. Notre périple devrait nous entraîner à repérer les éléments structuraux de l'originaire et de l'infini, c'est-à-dire de ce qui préexiste au début de la vie et de ce qui perdure au-delà de la mort. Nous voyons d'emblée que notre propos sera constitué d'hypothèses plus ou moins prospectives qui devraient permettre au lecteur d'y confronter ses propres conceptions élaborées à partir de l'interrogation concernant les limites de sa propre temporalité. En effet, tout humain construit de telles théories, et les humains ont élaboré de nombreuses conceptions des bornes de la temporalité au fil des différentes cultures et civilisations.

\section{De l'archaïque ${ }^{1}$}

Nous pourrions formuler l'hypothèse selon laquelle l'archaïque serait une construction défensive contre le temps initial de l'informe et l'impression de vide ressenti par le sujet émergeant du chaos initial (de Mijolla-Mellor, 
2005). C'est par la voie de la régression vers l'inconscient atemporel que nous pouvons rencontrer ce socle archaïque au niveau du sujet individuel. C'est dans la prise en compte du primitif résistant à l'historisation que nous pouvons le rencontrer au niveau sociétal. Nous pouvons le postuler à partir de ses effets d'après-coup qui conduisent à envisager une structure préexistante à l'actuelle.

Pour tenter de situer plus précisément l'archaïque, au niveau du sujet individuel, nous allons utiliser les effets d'après-coup rencontrés dans l'expérience tant théorique que pratique de la psychanalyse. Il s'agira de repérer sa persistance dans l'expérience de la psyché, et plus particulièrement dans les premiers temps de la vie de l'infans, donc de l'humain avant son appropriation du langage. Nous reprendrons pour ce faire les éléments d'un travail antérieur (Bonnet, 2002) dans lequel nous avions repéré la réactivation de l'archaïque dans les problématiques de guerre et de terrorisme.

Tout se passe comme si l'infans en venant au monde, se trouvait confronté à une expérience fondamentale faite de détresse et de déréliction ${ }^{2}$. Cet état premier est facile à se représenter, en prenant en considération le fait que le bébé, sorti du ventre maternel, se trouve propulsé dans un monde radicalement différent du monde antérieur qui l'a contenu et protégé durant les neuf mois précédant sa sortie dans le monde extérieur. Dans l'univers antérieur, postulons qu'il ait trouvé une satisfaction assez parfaite et totale de ses besoins de développement.

Dans cette confrontation au monde extérieur, il se trouverait donc dans une situation de déréliction, tant physiologique que psychologique, liée aux effets du manque renforcé par la confrontation à l'inconnu de l'informe. Ce manque fondamental est dû à une différence de contenance et de réponse aux besoins qui entraîne un ressenti d'insécurité maximale. Cet éprouvé est d'autant plus important que le petit homme se trouve dans l'incapacité de subvenir par lui-même à ses besoins fondamentaux pour pouvoir rester en vie. Nous remarquerons au passage que l'expérience de la mort pourrait être pressentie dans cet espace-temps inaugural du tout début de la vie ${ }^{3}$. Dans la plupart des cas, cette insécurité va se trouver, en partie, comblée par les soins, en particulier maternels, dont il va être l'objet, et qui sont indispensables à sa survie. Il a besoin, cet infans, loin de pouvoir s'exprimer dans le registre du langage, de cette autre maternelle qui va lui procurer la satisfaction de ses besoins premiers, où l'apport de nourriture et les soins corporels vont jouer un rôle primordial. Il existe donc un appel originaire à l'autre permettant l'émergence de l'état de détresse associée à la nécessaire satisfaction des 
besoins. L'appel à l'autre de la part de l'infans implique une réponse adaptée qui est, à proprement parler, strictement vitale. La détresse primordiale est une première expérience fondamentale, un socle, un point d'organisation et de structure, qui sera certes frappé d'oubli, objet de refoulement. Cet éprouvé de détresse pourra en tant que tel revenir, ou être plus ou moins confusément rappelé lorsque, dans sa vie ultérieure, le sujet humain aura à traverser d'autres expériences réactivant les conditions de déréliction. Nous pouvons faire l'hypothèse qu'une telle détresse est empreinte d'un éprouvé de terreur face au manque vital dont l'infans va se défendre en ayant recours au repli sur soi ou/et à l'étayage sur l'autre. Un tel étayage sera facilité par la présence d'une mère ou d'un substitut maternel efficace et bienveillant. Mais l'infans pourra toujours tenter de méconnaître ce recours originaire à l'altérité sous la forme d'un déni le conduisant à s'imaginer une capacité d'autosatisfaction narcissique qui ne viendrait que de lui seul et qui correspondrait au narcissisme originaire (Bonnet, 2001).

Le désir et l'activité de représentation concomitante vont se greffer sur ces expériences liées à la satisfaction des besoins fondamentaux, permettant le dépassement de l'état primordial de manque et de celui de la confrontation à l'informe qui engendrent la détresse. Le désir va s'exprimer soit comme tentative de retrouver cette rencontre avec l'autre, soit de la dénier suivant la modalité mégalomaniaque d'omnipotence, voire sous la forme d'une sorte de «désir de non-désir» considéré comme traduction de la pulsion de mort. L'expérience de satisfaction et de plaisir implique, outre l'activité désirante, celle de représentation. Il existe une propension chez l'infans à se figurer, puis à se représenter l'objet du besoin et l'autre, qui en est le transfuge.

Cette représentation originaire implique la capacité d'halluciner l'objet du besoin qui fait manque. C'est en effet en partie dans la capacité hallucinatoire de représentation de l'objet absent que l'infans peut vivre la nonsatisfaction immédiate de son besoin. Nous constatons que la représentation s'appuie tant sur le facteur lié à la perception de la réalité extérieure que sur la construction hallucinatoire du fantasme. L'expérience de besoin qui structure de façon concomitante celle du désir s'organise selon les modalités de «Prendre en soi» et de «Rejeter hors soi », pour reprendre la définition de toute représentation selon la théorie de Piera Aulagnier (1975). Prendre en soi, et conserver ce qui est considéré comme bon ou comme source de plaisir, et rejeter hors soi ce qui est mauvais car source de déplaisir. Les catégories du bon, du mauvais, et celles du soi et de l'étranger à soi, sont donc liées intrinsèquement aux expériences de plaisir et de déplaisir qui ont un sens 
spécifique du point de vue psychanalytique, dans la mesure même où ils sont les composants et les organisateurs du désir.

Nous nous proposons d'élargir maintenant notre compréhension de la problématique de l'archaïque en l'envisageant du point de vue sociétal. Les sociétés archaïques, entendues comme préhistoriques, préexistent à celles qui impliquent la direction d'un État. Edgar Morin (2001) définit avec précision la société archaïque comme étant le prototype des premières sociétés d'homo sapiens humaines, qui vivaient de chasse, de ramassage et de cueillette. Dans ce type de sociétés, il n'existait pas d'État, et c'est «la magie, le mythe et le rite qui sacralisent les règles d'organisation de la société» (p. 150). Morin note qu'il demeure « un noyau archaïque plus ou moins intégré dans toutes les sociétés ultérieures : le rôle générateur - régénérateur de la culture, le maintien, voire la résurgence de la division en bio-classes (hommes-femmes) et classes d'âge, les règles, normes et interdits du sexe, le mythe fraternitaire soudant la communauté.» (p. 151). Il paraît selon lui essentiel de prendre en compte au sein de toute société, le fait que chaque individu soit «à la fois un sujet égocentrique ${ }^{4}$ et un moment/élément d'un tout sociocentrique ${ }^{5}$ ( (p. 153). La relation individu/société est complémentaire et antagoniste, elle est donc «dialogique», pour reprendre le concept élaboré par E. Morin. Le «noyau archaïque est bien présent et donc actualisable et actualisé dans toute société historique; ainsi, le conflit et la fraternité, l'égocentrisme et l'altérité qu'impliquent le sociocentrisme, la barbarie et la culture, sont autant d'antagonismes que nous retrouverions présents et en dialogue tant chez l'individu que dans l'espace social.

E. Morin (2001) montre dans ses travaux la réalité selon laquelle l'homo sapiens n'est pas qu'empreint de sagesse, mais qu'il l'est aussi de folie et de démesure: il est homo demens c'est-à-dire démesuré. Il est donc composite, c'est-à-dire à la fois sage et démesuré, hésitant entre ces deux positions, trouvant des compromis entre elles: tel serait l'individu psychique. La cure analytique permet cette mise en évidence des compromis passés entre sagesse et folie par un sujet donné pour survivre à la fois psychiquement et socialement. Il semble que «le passage à la démesure constitue un moment caractéristique de destruction des sociétés archaïques autorégulées» (p. 117).

Retenons de notre parcours sur la piste de l'archaïque, qu'il se constitue comme réaction défensive au chaos originaire et qu'il se structure dans un espace-temps originaire, à la fois préhistorique, tout en étant marqué d'une valence maternelle. Le sujet individuel et social en émerge sur un mode composé de sagesse et de démesure, d'égocentrisme et de sociocentrisme, entre solipsisme et altérité. 
Piera Aulagnier (1975) a montré le paradigme de l'émergence de l'archaïque en postulant, dans l'activité de représentation, le pictogramme originaire du prendre en soi/rejeter hors soi, à partir des processus primaires du fantasme et des processus secondaires de l'idée. Pour Sophie de Mijolla (2005):

L'archaique pourrait donc être défini comme l'incarnation de survivances, répétition et actualisation d'un vécu fossile. Ces formes épurées, condensées dans des images ou des mots ont pour destin aussi bien de se répéter à l'identique dans des expériences oniriques ou dans le vécu de l'inquiétante étrangeté par exemple, que de s'élaborer en mythe. (p. 18)

Pour ma part, j'ai avancé dans certains de mes travaux antérieurs (2002, 2009, 2011, 2012), la possibilité d'envisager comme résultantes des formules de compromis pour émerger de l'archaïque, deux modalités auxquelles le sujet psychique pourrait avoir recours quand il est confronté à l'état de détresse face au chaos de l'informe. Ces deux modalités seraient à la fois des possibilités d'où leur nom de potentialités.

1. La potentialité mélancolique est une modalité possible de réponse à un éprouvé de manque par rapport à la réalité objective ou fantasmée d'une perte d'objet: elle se traduit par un repli égocentrique du sujet sur son Moi accompagné d'une perte de l'estime de soi par rapport aux autres ${ }^{6}$. Nous pouvons la comprendre comme une tentative plus ou moins désespérée de maintien de la confusion originaire entre le Moi et l'objet extérieur. Une des réactions, et en même temps une issue possible de cette position mélancolique, peut s'exprimer dans l'omnipotence mégalomaniaque qui signe le triomphalisme du narcissisme originaire jusque dans la mise en acte suicidaire. La propension mélancolique est aussi une des expressions possibles du masochisme originaire.

2. La potentialité paranoïaque s'organise à partir de la reconnaissance de l'objet extérieur. Ce dernier fait l'objet d'une différenciation avec le Moi qui le ressent comme insupportable parce qu'étranger. Cet objet cristallise sa haine et le Moi vise sa destruction. La propension paranoïaque s'organise sur la base d'un sadisme originaire qui, projeté sur l'objet, est lui-même réactionnel au masochisme originaire infiltrant la modalité mélancolique.

Ces deux potentialités principales fonctionnent comme modalités en impasse psychopathologique lorsque chacune d'entre elles devient systématique. Elles peuvent aussi s'allier selon des modalités diverses pour un sujet 
individuel donné, comme elles peuvent être stimulées, voire valorisées par l'ensemble social auquel appartient le sujet individuel. Nous aurons donc aussi à prendre en considération ces deux potentialités psychiques différentes lorsque nous tenterons d'expliciter les réactions face à la problématique de la mort, la sienne propre ou celle d'autrui.

Ces deux potentialités émergent du fond pervers du fonctionnement psychique au sens où Freud parlait de "prédisposition perverse polymorphe » (Freud, 1905). Nous pouvons envisager une formation de compromis originaire entre potentialité mélancolique et potentialité paranoïaque sous forme de perversion. En suivant cette voie régrédiente, nous sommes ainsi conduits à repérer les empreintes du masochisme et du sadisme à l'orée de la constitution du sujet psychique.

Retenons que «l'archaïque » se constitue sur le socle pervers de la psyché. Il s'organise suivant deux tendances antagonistes: l'une de solipsisme, l'autre d'altérité qui s'exprimeront et se conjugueront tout au long de la vie. Il y aurait donc dès l'origine un refus, un déni de réalité portant sur la nécessité de la présence de l'autre, au profit d'une pratique et d'un discours composés de propos d'autosuffisance, de complétude narcissique et d'évitement de l'autre. Les fondements des deux positions archaïques utilisées par le sujet que nous venons de distinguer, s'appuient sur l'articulation du narcissisme originaire au masochisme originaire.

Nous pourrions être tentés de comprendre la propension au repli solipsiste comme simple émergence d'un fondement narcissique. Ce pourrait être seulement une question d'image, comme l'implique l'eau limpide dans laquelle se mire Narcisse, teintée d'un auto-érotisme dans lequel se complaît le sujet. Comme nous l'avons écrit précédemment, il pourrait s'agir d'une modalité de narcissisme originaire. Nous pouvons aussi postuler l'exercice d'un contrat, autant imaginaire qu'implicite, passé avec un autre soi-même qui serait chargé de distiller les conditions de souffrance physique et morale qui vont être les conditions de l'accès au plaisir. De telles considérations viennent de nos travaux concernant la mélancolie (Bonnet, 1995, 2005). Cette notion de contrat interne s'opérerait dans la construction de la psyché entre deux parties dédoublées, voire clivées du Moi que nous retrouvons d'ailleurs dans le mythe de Narcisse. Ne serait-ce pas sur cette base de dédoublement de soi-même que l'autre pourrait commencer à se loger? L'autre pourrait être originellement compris comme partie émanant de soi, ou mieux comme partie créée par soi ; le passage du créé au trouvé-créé dont parle Winnicott (2010) signerait la capacité secondaire de prendre en compte l'altérité. 
Dans l'ère comme dans l'aire des débuts, narcissisme et masochisme feraient à la fois bon marché et bon ménage confusionnels. L'image primaire de soi se constituerait sur la base d'une souffrance de déréliction convertie en modalité de plaisir. Elle tendrait à exclure toute trace d'autre qui chercherait à se représenter, suivant ainsi la voie de la potentialité paranoïaque. Qui plus est, le Moi lui-même, en tant que représentant une trace du vivant, pourrait être aussi l'objet d'attaques visant sa disparition, ainsi que nous l'indique la modalité mélancolique; nous pourrions ainsi comprendre l'attitude de Narcisse face à sa propre image qui consiste à se noyer en croyant trouver une image idéale de lui-même. Nous sommes alors amenés à constater une grande proximité structurelle dans la constitution de la psyché, entre le masochisme originaire et son contre-investissement dans les termes du sadisme; le masochisme, en tant que manifestation originaire, est le constituant princeps de ce qu'il est convenu d'appeler «l'archaïque». Il se constitue comme étant à la fois une étape préœdipienne et prégénitale de la constitution psychique, tout en étant aussi un point d'appel permanent à un retour, voire recours en arrière possible, que le sujet a la possibilité d'utiliser pour se maintenir en vie psychique envers et contre tous. Ce retour ou ce recours ne pourrait-il pas aussi permettre une quasi expérience de la mort? Si le masochisme est constitué sur la base d'un mélange des pulsions sexuelle et de mort, nous pouvons remarquer la fragilité de cette intrication dans la mesure où la pulsion de mort dans ses effets éradiquants s'avère prompte à ramener le fonctionnement psychique à un point d'inertie proche de l'état létal du moi ou de l'objet, suivant la prédominance de l'impact masochiste ou de son contre-investissement sadique.

En résumé, le sujet est confronté à l'origine, à la prégnance d'un système archaïque de type pervers, empreint de masochisme et de sadisme, dont l'objectif commun vise le triomphe du solipsisme sur toute objectalité et qui plus est, sur toute prise en compte de l'altérité; cette visée solipsiste flirte dangereusement avec la destruction tant de soi que de l'autre. Ce socle archaïque de la constitution de la psyché, qui induit un «égocentrisme» fondamental, constitue un point d'appel permanent auquel le sujet socialisé, primarisé, voire même secondarisé pourra toujours avoir tendance ou tentation de faire recours pour s'y réfugier «solipsistement vôtre! " Mais, comme nous l'avons constaté, le sujet a dès l'origine besoin de l'autre pour survivre. Ainsi dès «l'archaïque», il se trouve aussi pris dans le registre de l'altérité, et qui plus est, il provient de l'Autre qui existe hors de lui. Le sujet psychique est donc constitué de deux tendances antagonistes qui demeurent en dialogue plus ou 
moins ouvert. Entre la tendance au repli solipsiste et l'ouverture vers l'altérité, le sujet historique se trouvera cependant en diverses situations à avoir à opérer des choix. Encore faudra-t-il, pour que le sujet psychique poursuive au mieux son chemin en temporalité, que vienne s'inscrire la référence à une position tierce qui lui permette de se déprendre de toute confusion avec le registre maternel. Cette référence implique dans le schéma œedipien canonique la fonction paternelle qui ouvre à la pleine altérité.

Edgar Morin (2001), dans l'ouvrage de synthèse de son œuvre, écrit:

Le sujet est égocentrique; mais l'égocentrisme ne conduit pas forcément à l'égoïsme [...] il y a dans la situation de sujet une possibilité égoïste qui va jusqu'à tout sacrifier à soi, et une possibilité altruiste qui va jusqu'au sacrifice de soi. La première peut mener à l'antagonisme à l'égard du semblable et à la limite au meurtre de Caïn. La seconde peut susciter une fraternité qui incite à donner sa vie pour l'ami, le frère [...]. La qualité de sujet porte en elle la mort de l'autre et l'amour de l'autre. (p. 66-67).

\section{De la mort}

Nous avons remarqué que l'expérience de la mort en tant que non vie pourrait être ressentie dans cet espace-temps inaugural du tout début de la vie psychique dont nous venons de parler. Le chemin de la vie qui s'ensuit mène à la mort dans une temporalité plus ou moins longue mais inéluctable. La mort qualifie l'état d'un organisme biologique ayant cessé de vivre, marquant ainsi la fin de la temporalité de la vie humaine. Cependant, au niveau biologique, le patrimoine génétique se perpétue dans la descendance et la vie se perpétue dans l'espèce et les écosystèmes tout en évoluant. Du point de vue philosophique et religieux, de nombreuses conceptions postulent l'hypothèse d'une vie psychique après la mort.

De toutes les façons, la fin de vie véhicule son cortège d'angoisses tout au long de la vie du sujet, ainsi que différentes attitudes devant la mort, qui se trouvent comprises aussi de façon différente selon les diverses cultures qui peuvent composer à un moment historique donné une civilisation. Actuellement, nous sommes en passe de nous demander si ce n'est pas la problématique du vieillissement qui devient plus inquiétante que celle de la mort. Vieillir, à l'heure actuelle, ne consiste pas à gagner en sagesse mais est plutôt vécu comme un signe de déchéance. La mort apprivoisée, selon Ariès (1983), finit quand la proximité entre le mort et vivant n'est plus tolérée. La mort serait désormais de plus en plus considérée comme une limite 
intolérable et non envisageable, intervenant comme rupture, alors qu'au Moyen Âge par exemple, elle était si présente au quotidien qu'elle faisait en quelque sorte partie de la vie. Edgar Morin (1976) développera le point de vue selon lequel à la différence de tout être vivant, l'homme nie la mort en développant des croyances en un Au-delà. Ainsi, les attitudes vis-à-vis de la mort comme les représentations de celle-ci vont se modifier au fil de l'histoire du sujet, comme elles l'ont été au fil de l'histoire des civilisations. Cependant Freud (1915) a soutenu l'idée selon laquelle la mort du sujet n'était pas représentable dans la mesure où le sujet ne pourrait envisager sa propre mort, persuadé qu'il serait dans l'inconscient de son immortalité.

Une telle assertion se discute, car nous pouvons nous demander si ce ne sont pas plutôt les phantasmes de mort qui sont refoulés et préservés sur l'autre scène de l'inconscient (Bonnet, 1991). Ce serait dans ce sens-là que l'on peut entendre que l'inconscient ne connaît ni la castration ni la mort. La cure analytique assurant la levée du refoulement des représentations de la mort ne permet-elle pas au sujet d'admettre son état d'être mortel? Il existe deux axes de compréhension de la mort dans l'œuvre de Freud: dans le registre œdipien, la mort est l'emblème de la castration et elle renvoie à la vie et à l'infantile, alors qu'à partir de 1920, la mort, c'est ce vers quoi retourne la vie, à savoir l'inerte ou l'inorganique qui la précède et qui en devient le but. Le retour vers l'inanimé, que nous pourrions aussi appeler l'informe, implique un recours à certaines modalités de l'archaïque dont nous avons parlé précédemment. Le but final serait de ramener ce qui vit à l'état inorganique et c'est pourquoi, à la suite de Freud, nous pouvons alors parler de pulsion de mort. Guy Rosolato (1978) a situé la mort dans La relation d'inconnu, en montrant qu'à partir de 1920, elle se trouve en quelque sorte intériorisée par Freud. L'intériorisation de l'impensable de la mort la délivre en quelque sorte d'une sujétion extérieure en la ramenant au cœur du narcissisme, ce qui rend pensable, pourrait-on dire, l'impensable de la mort. Notons que cet impensable de la mort se trouve à l'origine des angoisses les plus vives et par voie de conséquence, des défenses contre ces angoisses qui animent justement les représentations de la mort et les conceptions d'un au-delà de la mort. Le positionnement d'une pulsion de mort au niveau intrapsychique, ou plus précisément endopsychique rendrait ainsi possible le développement d'une pensée sur la mort.

Piera Aulagnier (1975) a repris le terme de Thanatos pour réunir en un seul vocable tant les forces de déliaison, celles de la haine ainsi que la contrainte interne poussant à la quiescence de la constance et de l'inertie, que 
les excitations externes et internes d'ordre sexuel ou non. Elle a montré comment la théorie de la pulsion de mort se situe à proximité des théories de l'archaïque. Thanatos, incluant la haine radicale vis-à-vis de tout objet interne, atténue le déplaisir de l'excitation, en laissant libre cours au plaisir d'autodestruction qui peut s'entendre comme première manifestation de la pulsion de mort. Nous voyons ainsi que cette haine radicale vise bel et bien l'existence de tout «hors soi», ainsi que nous l'avons vu précédemment lorsque nous envisagions les fondements de l'archaïque. Nous pouvons nous demander quelles peuvent être les résurgences d'une telle haine radicale lorsque le sujet se trouve confronté à l'imminence de la mort.

Nous avons souvent remarqué le travail de différenciation concernant les pulsions de mort opéré par Nathalie Zaltzman (1986). Si certaines d'entre elles sont liées, intriquées, d'autres se traduisent sous forme de « décharge meurtrière (accomplie ou fantasmatique) qui procure une jubilation, une extase d'investissement irréductible à nos repères actuels. » Retenons de ses travaux la distinction entre «la possibilité de se détacher de l'objet et la capacité de l'aimer. » Le détachement vis-à-vis de l'objet n'est pas seulement une activité de déplacement de l'investissement, il nécessite d'abord agressivité, intensité de destruction, vœu de mort, pour que cet objet perde de son importance. Il s'agit dans cette conception d'énergies qui ont des fonctions aussi nécessaires dans l'économie psychique que l'amour, la sollicitude, la réparation. Ainsi, nous pourrions envisager que la haine et l'amour assument des fonctions tout aussi importantes dans l'économie psychique. Le désir de mort, articulé au désir de meurtre qui a animé certains moments de la vie du sujet, ne pourrait-il pas se réactiver au moment ultime pour permettre le passage de la vie à la mort?

Cette modalité de passage vers la mort ne coüncide pas vraiment avec l'idée d'un sujet réconcilié avec lui-même "selon un niveau de ré/intrication pulsionnelle assez bon » selon les termes employés par Benno Rosenberg (1991), à propos du travail de mélancolie. L'objectif serait pour lui de lier la pulsion de destruction par l'Éros, permettant ainsi de juguler la haine de l'objet dont l'augmentation a provoqué l'accès mélancolique. Il est question pour Benno Rosenberg dans l'issue du travail de mélancolie de permettre les « retrouvailles » avec l'objet sur un mode masochiste plutôt que d'opérer un deuil psychique qui impliquerait le meurtre en vue de la destruction de l'objet. Ne pourrait-il pas aussi s'agir d'une autre modalité d'envisager le passage vers la mort?

Nous pouvons envisager la nécessité d'accompagnement du mourant comme correspondance ultime de l'appel à l'exercice de la violence maternelle 
secondaire, dont parle Piera Aulagnier, venant donner sens aux ultimes éprouvés corporels. Dans la même direction de sens, rappelons-nous les trois coffrets (Freud, 1913) mettant en scène les trois images de la femme-mère, et plus particulièrement celle «de la silencieuse déesse de la mort» recueillant l'homme en fin de vie. En résumé, la mort constitue un événement psychique particulier rencontré dès l'archaïque jusqu'à sa survenue dans le réel. La problématique de la mort à venir accompagne le sujet psychique tout au long du parcours de temporalité. Prenant en compte la deuxième topique freudienne, constatons la conflictualité qui se dessine entre le Moi, qui à la fois sait et ne veut rien savoir de la mort qui se profile à l'horizon, et le Ça qui continue à désirer du fait de l'efficience de la libido ${ }^{7}$.

Confronté à la problématique de la mort, nous sommes en droit aussi de nous poser la question récurrente au fil des siècles à savoir celle de l'Au-delà. Cette question fait controverse au cœur même de toutes les civilisations ainsi que dans l'élaboration des mythes magico sexuels (de Mijolla-Mellor, 2002) par chaque sujet psychique, dans lesquels la problématique de la mort s'articule à celle du meurtre. La problématique de l'Au-delà, comme je l'ai travaillée récemment (Bonnet, 2013) introduit un dialogue entre le destin individuel de psyché (âme) dans son au-delà du corps mort avec une conception de dieu(x) ou de Dieu. Une telle orientation vient se poser en termes de transcendance par rapport à l'humain. Sans entrer dans la problématique essentielle de l'existence de Dieu et celles concomitantes de Foi ou de croyance, je me bornerai à quelques repères concernant le projet identificatoire du sujet qui illustre de façon plus laïque la traversée de la temporalité.

\section{Du projet identificatoire}

L'existence du projet identificatoire assure la pérennité de la traversée de la temporalité; il se constitue dans la période historique de la structuration de la psyché, succédant à l'archaïque et se poursuivant jusqu'à la mort du corps biologique. Il s'organise sur la base de la constitution de ce que nous avons travaillé autour de l'identification symbolique (Bonnet, 1999). Nous avons établi un lien entre la problématique de l'identification symbolique et une des conceptions de Freud (1923) concernant l'identification primaire, à savoir celle qu'il introduit dans Le moi et le ça dans les termes de l'identification au père de la préhistoire personnelle, identification directe, immédiate, plus précoce que tout investissement d'objet. Nous sommes donc confrontés à l'introduction d'une identification fondamentale, primaire au sens de primordiale, préexistante à la constitution du sujet psychique, qui peut s'entendre 
comme la reprise ontogénétique de l'identification originaire au père de la horde primitive (Freud, 1912-1913).

Piera Aulagnier (1975) a traité de l'identification symbolique en termes d'espace:

L'espace où le «Je » doit advenir, qui est aussi le seul espace où il peut advenir; son organisation est placée sous l'égide d'une série de signes linguistiques: ceux propres à l'affect et ceux propres au système de parenté - qui, en nommant une chose ou un élément définissent la relation présente entre l'objet qu'on nomme et celui qui s'approprie et énonce cette nomination. (p. 169)

L'identification symbolique est organisatrice de la structure identitaire de tout sujet psychique. Ses fondements reposent sur les deux interdits fondamentaux: l'interdit de l'inceste et l'interdit de meurtre. Sa fonction consiste donc à organiser les positions identificatoires en proscrivant certaines réalisations pulsionnelles incompatibles avec les exigences culturelles dont la transgression entraînerait un risque de désidentification.

L'identification symbolique est un des éléments de l'identification primaire. Il s'agit d'un ensemble identifié et donc identifiable qui émerge d'un registre transcendant transmis par le registre sociétal; l'identification narcissique constitue sur la base de l'archaïque l'autre composant de l'identification primaire. "Le projet identificatoire» apparaît alors comme une problématique de tension interne au sujet psychique entre l'identification symbolique à l'Autre et l'identification narcissique à soi-même. Ainsi, du fait du recours possible à chaque pôle du projet identificatoire, mais aussi du fait de la tension existant entre les deux pôles, la quête identificatoire demeure pérenne dans la temporalité au moins jusqu'au point de mort psychique qui ne correspond pas forcément avec la mort biologique. La tension interactive entre une tendance conservatrice de l'objet selon la modalité de l'identification narcissique, et une tendance à l'idéalisation sublimatoire selon le modèle de l'identification symbolique, accompagnera tout sujet psychique tout au long de sa rencontre des événements qui jalonnent son parcours, et son engagement dans la temporalité s'en trouvera forcément conflictuel.

\section{Conclusion}

Tout irait donc pour le mieux de l'archaïque à la mort dans un monde tant social qu'individuel où le sujet psychique fonctionnerait en se situant 
entre les deux pôles du projet identificatoire. Un tel cheminement relève de l'idéal ou d'une certaine normalité névrotique comprise dans la conflictualité œdipienne et la castration symbolique. Il est battu en brèche par la problématique psychotique liée à l'exacerbation de l'identification narcissique et à l'évanescence de l'identification symbolique. Qui plus est, d'autres modalités de fonctionnement psychique sont apparues dans les termes des états limites, des pathologies narcissiques et dans ceux de la perversion. Elles sont la traduction de la crise actuelle de la subjectivation elle-même, dans la mesure où le sujet a perdu ses points de repère marqués antérieurement du signe de la fonction patriarcale actuellement en voie de dissolution, laquelle était imprégnée d'identification symbolique. Nous sommes conduits à nous demander si de tels sujets actuels ne sont pas en train de devenir des enfants sans nom, des néosujets ${ }^{8}$ sans pouvoir faire recours ou avoir recours aux mots de l'Autre. De tels sujets sont des illustrations d'un sujet psychique en train de se constituer dans la quête de jouissance massive et permanente de tout objet, sans référence aucune à une quelconque identification symbolique.

Nous voyons aussi comment, dans un monde où la tiercéité est mise à mal, le corps peut devenir la limite vitale dans la mesure où il est le lieu ultime qui exprime justement la limite.

La maladie du corps peut devenir alors une modalité de réinvestissement pour tenter de déplacer la limite ultime. Les repères transmis par la tradition sont aujourd'hui pulvérisés dans la société postmoderne. Une telle pulvérisation nous oblige à reconsidérer tant la mort, la vie, le sexe, la naissance, la filiation, les modalités de transmission. Nous pouvons envisager que la mort fasse de moins en moins l'objet de déni mais qu'elle soit elle-même réinvestie à travers l'expression de nouveaux rites funéraires tels que nous pouvons déjà les découvrir dans les cérémonies laïques de recueillement et de paroles précédant la crémation par exemple.

Les modalités représentatives de l'identification symbolique sont mises en question du fait de la dissolution actuelle de la fonction patriarcale qui fut longtemps confondue avec elle. La valence religieuse a été tout d'abord remplacée par la valence scientifique qui a exercé pendant un temps une référence tierce. J'ai proposé récemment le constat suivant (Bonnet, 2012):

Actuellement, la société libérale de type marchand a promu la consommation immédiate s'appuyant sur la libération immédiate des pulsions de vie comme de mort. La société libérale consacre la fabrique d'individus mus par leurs pulsions que le système économique exploite et manipule. Le sujet 
postmoderne ainsi modelé présente des difficultés à accéder à la décision libre d'un sujet susceptible de maîtriser ses pulsions. La dérégulation actuelle de l'économie financière peut être considérée comme un des effets mortiferes du travail des pulsions au niveau de la culture. Elle peut avoir des effets de dérégulation de l'économie symbolique (disparition de l'autorité du pacte social) comme de l'économie sémiotique (apparition d'une novlangue libérale). Le sujet tant singulier que collectif se trouve pris dans un mouvement de désinstitutionalisation généralisée sur fond d'une perte de références symboliques (p. 241).

Nous pouvons alors effectivement nous demander si le progrès n'entraîne pas l'humanité vers la destruction radicale de l'espace humain. Serions-nous cette fois vraiment à l'aube de l'Apocalypse et d'un jugement dernier et définitif? Peut-on parler de régression individuelle et collective vers des modalités archaïques de vie sociale ou, plus exactement, vers des modalités intersubjectives qui auraient eu lieu à l'origine de la vie sociale et se réactualiseraient à temps donné? Faudrait-il admettre que ces différentes positions des acteurs sociaux existeraient de façon latente et cachée chez tout un chacun? La spiritualité impliquant la transcendance ne constituerait-elle pas un rempart à la régression en introduisant de nouvelles formes de tiercéité?

\author{
Marc Bonnet \\ 43520 Le Mazet Saint-Voy \\ bonnet.marc@wanadoo.fr
}

\title{
Notes
}

1. Le terme "archaïque» vient du mot grec arkhè qui signifie l'origine, le commencement mais aussi le principe, le fondement.

2. Traduction admise du terme allemand: Hilflosigkeit, traduit en termes de «désaide» en particulier dans les Euvres complètes de Freud publiées aux PUF.

3. Nous pouvons nous référer dans ce sens à l'article de Winnicott: La crainte de l'effondrement.

4. Souligné par nous.

5. Ibid.

6. Nous renvoyons le lecteur à un précédent travail: Bonnet, 1995.

7. Voir à ce sujet Dayan (1990) et De M'Uzan (1977).

8. Pour reprendre le terme de Jean-Pierre Lebrun, in La perversion ordinaire: vivre ensemble sans autrui, Denoël, 2007. 


\section{Références}

ARIÈS, P., 1983, Images de l'homme devant la mort, Paris, Seuil.

BONNET, M., 1991, Représentations infantiles du mort, Topique, nº 49.

BONNET, M., 1995, Prototypes identificatoires dans le travail de mélancolie, Topique, n 56.

BONNET, M., 1999, La double référence de l'identification primaire, Topique, $\mathrm{n}^{\circ}$ 69, 107-116.

Bonnet, M., 2001, On prend en charge un enfant. Prélude fantasmatique de la cure analytique. Topique, $\mathrm{n}^{\circ} 77$.

BONNET, M., 2002, Les Victoires de «l'archaïque», Topique, nº 81, 37-53.

BONNET, M., 2005, La mélancolie,http://www.quatrieme-groupe.org/pdf/Marc_Bonnet_ La_Melancolie_2005-03-19.pdf.

BONNET, M., 2009, Les variations de sens de la technique analytique, Topique, $\mathrm{n}^{\circ}$ 106, 49-68.

BONNET, M., 2011, Dialogue entre objectivation et subjectivation dans la clinique de la folie, Coq-Héron, n' ${ }^{\circ} 206,80-97$.

BONNET, M., 2012, De nouveaux processus de subjectivation, in La situation de la psychanalyse, Paris, In Press.

BONNET, M. 2013, De quelques avatars des rapports complexes entre Psyché et Dieu, À parâ̂tre.

CASTORIADIS-AULAGNIER, P., 1975, La violence de l'interprétation, Paris, PUF.

DAYAN, M., 1990, Déni de la mort et passage du temps, Psychanalyse à l'université, 57, 3-22.

DE MIJOLLA-MELLOR, S., 2002, Les mythes magico-sexuels dans l'imaginaire de l'enfant, Imaginaire et inconscient, 7 (3), 61-70.

DE MIJOLLA-MELLOR, S., 2005, De l'informe à l'archaïque, Recherches en psychanalyse, 3 (1), 7-19.

DE M'UZAN, M., 1977, Le travail du trépas, in De l'art à la mort, Paris, Gallimard.

FREUD, S., 1905, Trois essais sur la théorie de la vie sexuelle, Paris, Gallimard, 1989.

FREUD, S., 1912-1913, Totem et tabou, Paris, Gallimard, 1993.

FREUD, S., 1913, Le thème des trois coffrets, in Essais de psychanalyse appliquée, Paris, Gallimard, 1933/1971

FREUD, S., 1915, Considérations actuelles sur la guerre et la mort, in Essais de psychanalyse, Paris, Payot, 1968.

FREUD, S., 1923, Le moi et le ça, in Essais de psychanalyse, Payot, 1983.

LEBRUN, J-P., 2007, La perversion ordinaire: vivre ensemble sans autrui, Paris, Denoël.

MORIN, E., 1976, L'homme et la mort, Paris, Seuil.

MORIN, E., 2001, L'identité humaine, Paris, Seuil.

ROSENBERG, B., 1991, Le travail de mélancolie, in Masochisme mortifere et masochisme gardien de la vie, Pairs, PUF, 92-122.

ROSOLATO, G., 1978, La relation d'inconnu, Paris, Gallimard.

WINNICOTT, D. W., 1975, La crainte de l'effondrement, Nouvelle Revue de psychanalyse, 11, 35-44.

WINNICOTT D. W., 2010, Les objets transitionnels, Paris, Payot.

ZALTZMAN N., 1986, Baiser la mort? Une sexualité mélancolique, in De la guérison psychanalytique, Paris, PUF, 1998, 157-183. 